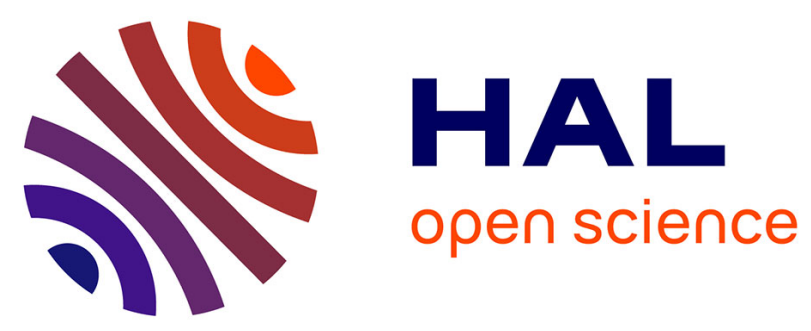

\title{
The GALAXIES inelastic hard X-ray scattering end-station at Synchrotron SOLEIL
}

J. M. Ablett, D. Prieur, D. Céolin, B. Lassalle-Kaiser, B. Lebert, M. Sauvage, Th. Moreno, S. Bac, V. Balédent, A. Ovono, et al.

\section{- To cite this version:}

J. M. Ablett, D. Prieur, D. Céolin, B. Lassalle-Kaiser, B. Lebert, et al.. The GALAXIES inelastic hard X-ray scattering end-station at Synchrotron SOLEIL. Journal of Synchrotron Radiation, 2019, 26 (1), pp.263-271. 10.1107/S160057751801559X . hal-02315437

\section{HAL Id: hal-02315437 \\ https://hal.sorbonne-universite.fr/hal-02315437}

Submitted on 14 Oct 2019

HAL is a multi-disciplinary open access archive for the deposit and dissemination of scientific research documents, whether they are published or not. The documents may come from teaching and research institutions in France or abroad, or from public or private research centers.
L'archive ouverte pluridisciplinaire HAL, est destinée au dépôt et à la diffusion de documents scientifiques de niveau recherche, publiés ou non, émanant des établissements d'enseignement et de recherche français ou étrangers, des laboratoires publics ou privés. 


\title{
The GALAXIES inelastic hard X-ray scattering end-station at Synchrotron SOLEIL
}

\author{
J. M. Ablett, ${ }^{\mathrm{a} *}$ D. Prieur, ${ }^{\mathrm{a}}$ D. Céolin, ${ }^{\mathrm{a}}$ B. Lassalle-Kaiser, ${ }^{\mathrm{a}}$ B. Lebert, ${ }^{\mathrm{a}, \mathrm{b}}$ M. Sauvage, ${ }^{\mathrm{a}}$ \\ Th. Moreno, ${ }^{\text {a }}$ S. Bac, ${ }^{\text {a }}$ V. Balédent, ${ }^{\mathrm{c}}$ A. Ovono, ${ }^{\text {d } M . ~ M o r a n d, ~}{ }^{\mathrm{b}}$ F. Gélebart, ${ }^{\mathrm{b}}$ \\ A. Shukla ${ }^{b}$ and J.-P. Rueff ${ }^{a, e}$ \\ aSynchrotron SOLEIL, L'Orme des Merisiers, BP 48, Saint Aubin, 91192 Gif-sur-Yvette, France, 'bnstitut de Minéralogie, \\ de Physique des Matériaux et de Cosmochimie, Sorbonne Université, UMR CNRS 7590, MNHN, IRD UMR 206, 4 Place \\ Jussieu, F-75005 Paris, France, 'Laboratoire de Physique des Solides, 91400 Orsay, France, dÉcole Nationale Supérieure \\ d'Ingénieurs de Limoges, France, and ${ }^{\mathbf{e}}$ Sorbonne Université, CNRS, Laboratoire de Chimie Physique - Matiére et \\ Rayonnement, LCPMR, F-75005 Paris, France. *Correspondence e-mail: james.ablett@synchrotron-soleil.fr
}

GALAXIES is an in-vacuum undulator hard X-ray micro-focused beamline dedicated to the study of the electronic structure of materials with high energy resolution using both photoelectron spectroscopy and inelastic X-ray scattering and under both non-resonant (NR-IXS) and resonant (RIXS) conditions. Due to the penetrating power of hard X-rays and the 'photon-in/photon-out' technique, the sample environment is not a limitation. Materials under extreme conditions, for example in diamond anvil cells or catalysis chambers, thus constitute a major research direction. Here, the design and performance of the inelastic X-ray scattering end-station that operates in the energy range from $\sim 4 \mathrm{keV}$ up to $12 \mathrm{keV}$ is reported, and its capabilities are highlighted using a selection of data taken from recently performed experiments. The ability to scan 'on the fly' the incident and scattered/emitted X-ray energies, and the sample position enables fast data collection and high experimental throughput. A diamond X-ray transmission phase retarder, which can be used to generate circularly polarized light, will also be discussed in the light of the recent RIXSMCD approach.

\section{Introduction}

Inelastic X-ray scattering (IXS) using hard X-rays ( $\geq 3 \mathrm{keV}$ ) is continuing to develop into a well established spectroscopic tool. Insertion device development, advancements in singlecrystal analysers and improved detector technology have been the impetus in developing IXS facilities at third-generation synchrotron radiation sources over recent years. In the hard $\mathrm{X}$-ray range, IXS instruments come in two flavours: very high resolution instruments with $\sim 1 \mathrm{meV}$ resolution for phonon excitations, and medium-resolution instruments $(\sim 100 \mathrm{meV}$ to $1 \mathrm{eV}$ ) mostly employed for resonant IXS, X-ray Raman scattering or X-ray emission. Examples of medium-resolution state-of-the-art hard X-ray IXS instruments can be found at

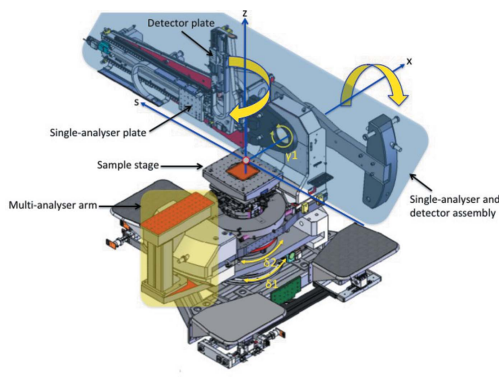
the European Synchrotron Radiation Facility (Hazemann et al., 2009; Llorens et al., 2012; Verbeni et al., 2009; Huotari et al., 2017; Moretti Sala et al., 2018; Glatzel et al., 2009a), the Advanced Photon Source (Fister et al., 2006; Xiao et al., 2016), the Stanford Synchrotron Radiation Light Source (Sokaras et al., 2012, 2013), SPring-8 (Ishii et al., 2013), the Diamond Light Source (Diaz-Moreno et al., 2018), the Swiss Light Source (Kleymenov et al., 2011), PETRA-III (Ketenoglu et al., 2018; Sundermann et al., 2017) and the Shanghai Synchrotron Radiation Facility (Ni et al., 2018; Duan et al., 2016; Gao et al., 2013). Spectrometers based on cylindrically bent crystal 
analyzers using the Von Hamos geometry, and which are particularly suited to IXS at X-ray free-electron lasers, are also becoming more prevelent (Hoszowska \& Dousse, 2004; Alonso-Mori et al., 2012; Szlachetko et al., 2017).

The various IXS processes (resonant and non-resonant) can be divided into several distinct techniques such as X-ray emission spectroscopy (XES), high-energy-resolved fluorescence detection (HERFD) and X-ray Raman spectroscopy (XRS). These often require very different experimental demands in terms of resolution and apparatus and there are many excellent reviews of these from both experimental (Rueff, 2009; Rueff \& Shukla, 2010, 2013; Rovezzi \& Glatzel, 2014; Glatzel \& Bergmann, 2005; Hayashi, 2013; Glatzel et al., 2009b) and theoretical (Schülke, 1986, 2007; van Veenendaal, 2014; Ament et al., 2011; De Groot \& Kotani, 2008) points of view. We have recently reported on the overall characteristics of the GALAXIES beamline (Rueff et al., 2015a) and also on the hard X-ray photoelectron spectroscopy (HAXPES) endstation (Céolin et al., 2013). This article completes the description of our beamline facilities.

\subsection{Major components of the GALAXIES beamline}

In this section, we describe the important aspects of the GALAXIES beamline that are relevant to the operation of the IXS end-station. The source consists of a $20 \mathrm{~mm}$-period invacuum $\mathrm{Nd}_{2} \mathrm{Fe}_{14} \mathrm{~B}$ undulator (98 periods, $1.96 \mathrm{~m}$ long) with a peak magnetic field of $1.04 \mathrm{~T}$ at a minimum gap of $5.5 \mathrm{~mm}$. A schematic of the optical layout of the beamline is shown in Fig. 1, where all X-ray mirrors operate at a grazing incidence angle of $5 \mathrm{mrad}$.

The first major optical component of the beamline is the silicon (111) liquid-nitrogen-cooled fixed-exit double-crystal monochromator (DCM) that provides an energy resolution of $\delta E / E \simeq 1.4 \times 10^{-4} \mathrm{FWHM}$. Higher energy resolution can be obtained with the DCM using the third- and fourth-order harmonics starting from $6.9 \mathrm{keV}$ and $9.2 \mathrm{keV}$, respectively. Circular polarization can be generated with the diamond $\mathrm{X}$-ray phase retarder (XPR), which is currently installed immediately downstream of the DCM (see Section 7). X-rays are then reflected by the M1 spherical collimating mirror, that provides harmonic rejection using selectable carbon or palladium coatings. For high energy resolution, a four-bounce high-resolution monochromator (HRM) can be inserted into the beam delivering $\delta E \leq 100 \mathrm{meV}$ FWHM bandwidth over the entire operating range for IXS measurements (Ablett et al., 2013). The HRM is currently undergoing commissioning, and high-resolution measurements performed so far have relied on the higher-order harmonics of the DCM. Two focusing modes are available at the IXS experimental endstation (Fig. 1): with the M2A toroidal mirror, the X-rays are focused onto the HAXPES experimental end-station that acts as a virtual X-ray source for the IXS KB focusing mirrors (equipped with rhodium and boron carbide coatings) to provide a micro-focused beam of $\sim 5 \mu \mathrm{m} \times 5 \mu \mathrm{m}$ FWHM. With M2A withdrawn from the beam, the beam is focused by the M2B toroidal mirror that gives higher X-ray intensity compared with the micro-focused mode (about a factor of three more due to the acceptance of the KB mirrors and reflectivity) but with a larger beam size of $30 \mu \mathrm{m}$ (vertical) $\times$ $90 \mu \mathrm{m}$ (horizontal) FWHM. The IXS end-station is separated from the beamline vacuum by a $50 \mu \mathrm{m}$ thin beryllium window, thus ensuring minimum absorption loss at low $\mathrm{X}$-ray energies. In order to provide an accurate estimation of the photon intensity delivered for planning a particular experiment, a measurement of the X-ray flux delivered to the IXS endstation at the sample position, with the DCM and M2B toroidal mirror, is shown in Fig. 2. These measurements were made using a calibrated $104 \mu \mathrm{m}$-thick silicon photodiode and corrected for absorption effects including air paths, detector efficiency and at a storage ring current of $430 \mathrm{~mA}$.

\section{The GALAXIES IXS spectrometers}

The GALAXIES IXS spectrometers are two integrated analyser systems that share the same support base and sample stage positioners. Both operate in the vertical scattering plane, use $10 \mathrm{~cm}$-diameter spherical analyser crystals with Bragg angles from $70^{\circ}$ to $89^{\circ}$ in the Rowland circle geometry and use continuous 'on-the-fly' scanning. The single-axis spectrometer,

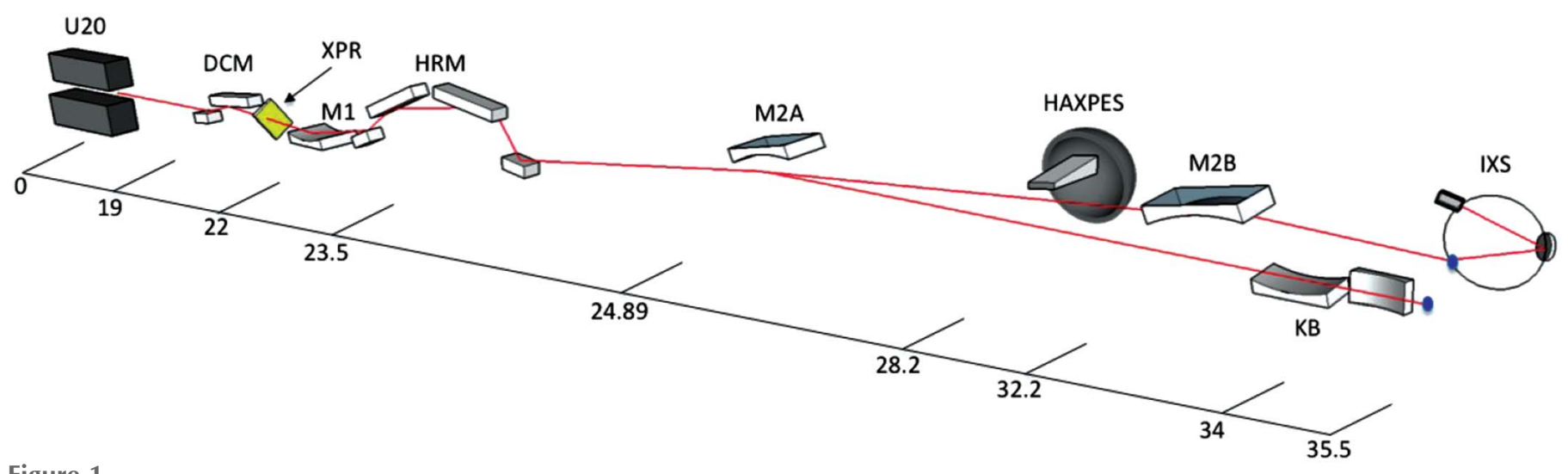

Figure 1

Side-view schematic of the optical layout for the IXS end-station. Beam propagation is from left to right and distances are in metres from the centre of the U20 undulator. Beamline apertures and X-ray beam diagnostics such as beam-position monitors and imagers are not shown for clarity. Refer to the main text for details on the abbreviations used. 


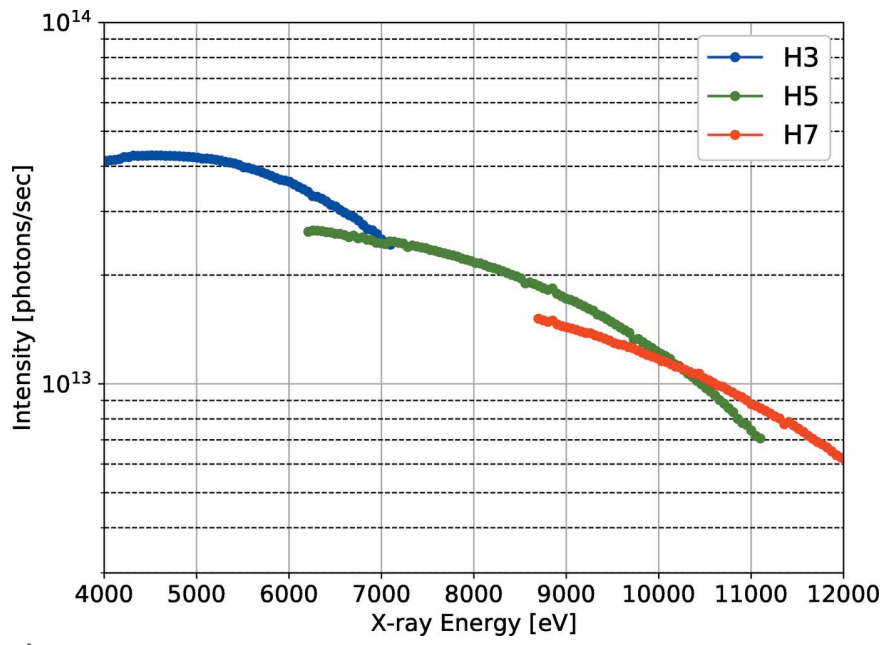

Figure 2

$\mathrm{X}$-ray intensity measured between 4 and $12 \mathrm{keV}$ at the IXS end-station sample position for the third (H3), fifth (H5) and seventh (H7) undulator harmonics at a ring current of $430 \mathrm{~mA}$, using the silicon (111) DCM and the M2B focusing mode. In the KB focusing mode, the X-ray intensity is a factor of three less than these values (see Section 1.1).

support base, multi-analyser arm and sample positioning stages were built by the Newport company. All stages have around $1 \mu \mathrm{m}$ or 1 milli-degree of translation or rotational resolutions, respectively, and a schematic of the spectrometer is shown in Fig. 3. The spectrometer sits on a high-precision five-axis motorized base using stepper motors that allows the spectrometer to be aligned accurately with respect to the incident beam position. The base rests on anti-skid antivibrational pads which ensure excellent vibration damping. The six-axis sample stage, which can accommodate loads of up to $150 \mathrm{~kg}$, allows for in-plane $x-s$ translations $( \pm 25 \mathrm{~mm})$ and height adjustments $( \pm 100 \mathrm{~mm})$. A horizontal rotation stage $\left( \pm 180^{\circ}\right)$ is mounted beneath these translation stages, under-

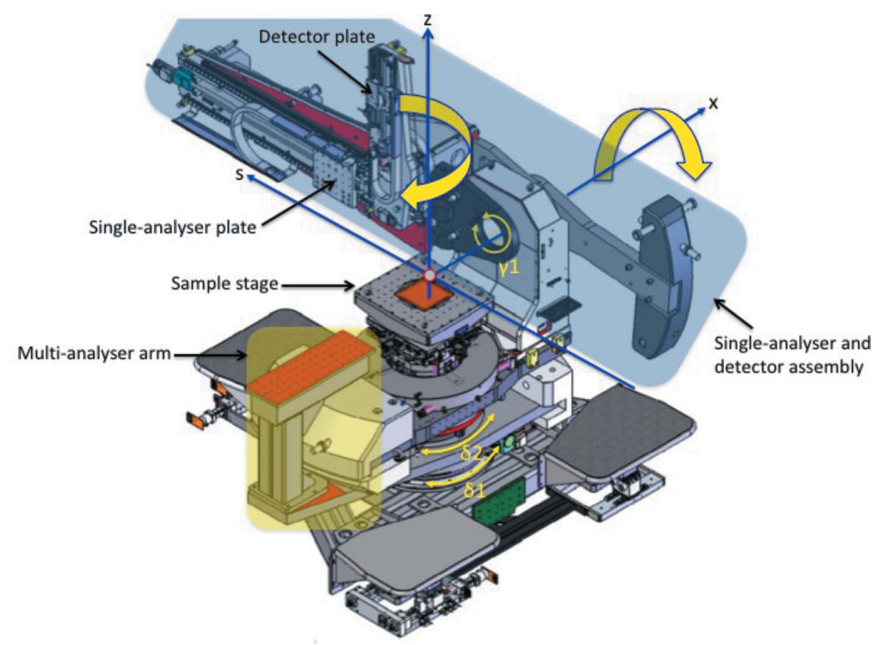

Figure 3

Schematic of the GALAXIES RIXS spectrometer. Two principal rotations, $\gamma 1$ and $\delta 1$, rotate the single-analyser and detector assembly in the vertical and horizontal planes, respectively. The multi-analyser arm can be rotated around the vertical axis by the $\delta 2$ rotation. The direction of incident X-rays is along the $s$-axis. neath which are located two concentric goniometer cradles $\left( \pm 10^{\circ}\right)$. For certain experiments, a sample support comprising a compact horizontal rotation stage, small manual goniometer and a helium/rough-vacuum environment is available for users. For all experiments and in order to minimize absorption losses, helium flight paths are used to eliminate air paths in the set-up.

\subsection{Single-analyser spectrometer}

The single-analyser spectrometer uses analysers with between $1 \mathrm{~m}$ and $2 \mathrm{~m}$ radii of curvature. It is optimized for high-resolution studies in both energy and momentum space and allows for a wide range of scattering geometries. The scattering angle can be varied in both the horizontal and vertical planes by the use of the $\delta 1$ and $\gamma 1$ rotations, respectively (Fig. 3). The analyser and detector are positioned on the Rowland circle by the use of five DC servo-motor-driven high-precision translation and rotation stages. Fig. 4 shows a photograph of this set-up superimposed with the Rowland circle geometry.

\subsection{Multi-analyser spectrometer}

The multi-analyser spectrometer uses four $10 \mathrm{~cm}$-diameter crystal analysers with radii of curvature of $0.5 \mathrm{~m}$ or $1 \mathrm{~m}$ and was designed in collaboration with and fabricated at IMPMCSU (Institut de Minéralogie, Physique des Matériaux et Cosmochimie, Sorbonne Université) (Gélebart et al., 2007). The multi-analyser system is fixed onto the short horizontal arm of the spectrometer (Fig. 3), and can be easily removed when required. For RIXS experiments, where the emitted energy is scanned on-the-fly, the configuration is that shown on the right-hand side of Fig. 5. In this configuration, the scan of the emitted energy is realised by changing the height of the

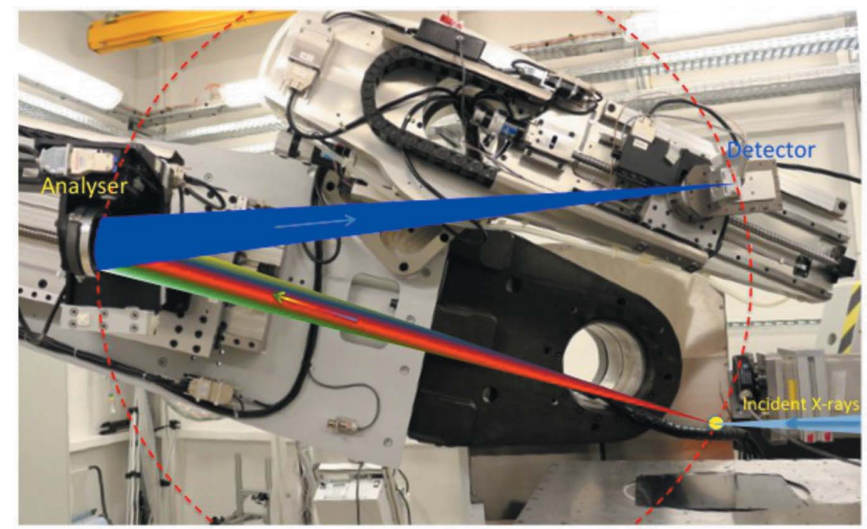

Figure 4

Photograph of the IXS single-analyser spectrometer superimposed with the Rowland circle geometry (represented by the dashed red circle). Monochromatic X-rays are focused at the sample and the scattered and fluorescence X-rays are collected by the analyser crystal that selects a narrow energy band of these X-rays which are then focused at the detector position. The spectrometer energy is scanned by varying the Bragg angle of the analyser whilst maintaining the Rowland circle geometry. Note that a helium flight path, which reduces the effect of air absorption, is omitted from the photograph for clarity. 

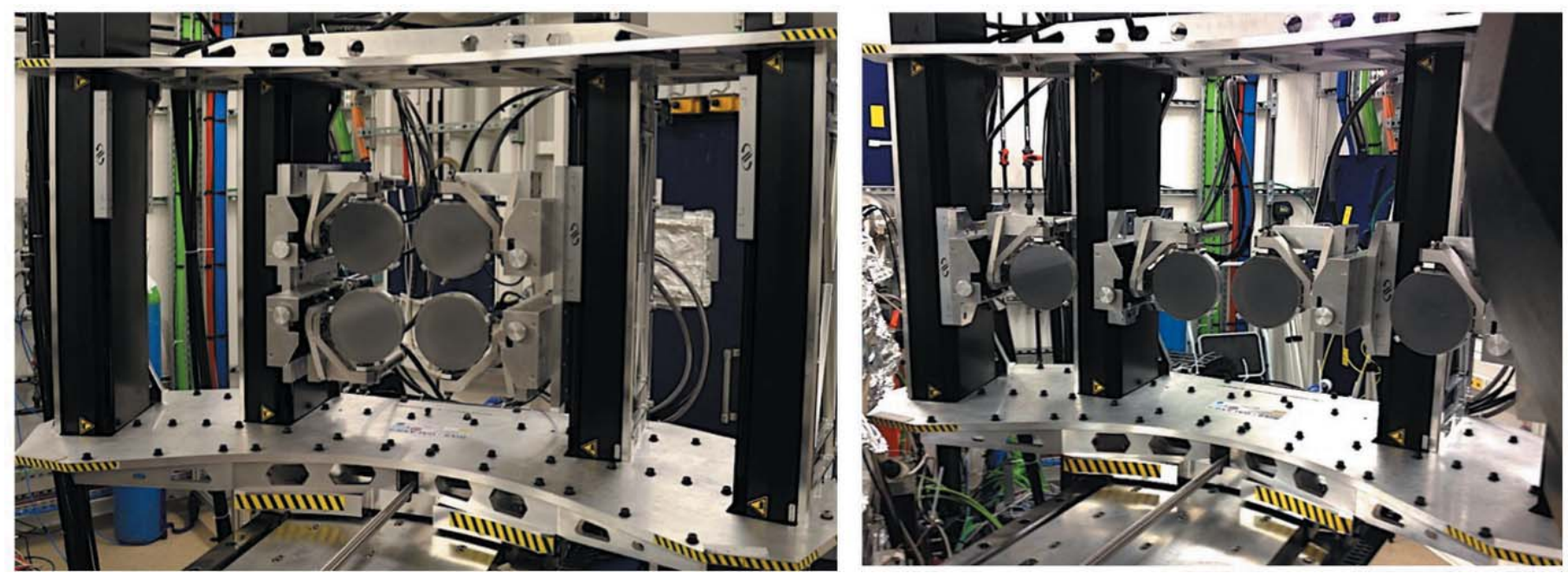

Figure 5

The multi-analyser arranged in the IXS (non-resonant/HERFD $2 \times 2)$ configuration (left) and in the scanning $(4 \times 1)$ configuration $($ right).

analysers while adjusting the analyser-sample and detectorsample distances to maintain the Rowland circle condition. For non-resonant IXS or HERFD measurements, where only the incident X-ray energy is scanned and the Bragg angle of the analyser crystals remains fixed, we typically use a $2 \times 2$ analyser configuration in order to increase the momentum resolution and provide a more compact arrangement (lefthand side of Fig. 5), and has been used for XRS measurements on $\mathrm{CeCuGe}_{2}$ (Rueff et al., 2015b), lithium borate glasses (Lelong et al., 2014, 2017) and ancient materials (Gueriau et al., 2017). Each analyser crystal is contained in a module that comprises highly rigid spring-loaded gimble mounts that are mounted onto a $50 \mathrm{~mm}$-long linear high-precision translation. Each module is then mounted on a $30 \mathrm{~cm}$-long vertical translation stage that allows the Bragg angle to be selected from $75.6^{\circ}$ to $90^{\circ}$ for $1 \mathrm{~m}$-radius analysers and covers $70^{\circ}$ to $90^{\circ}$ for $0.5 \mathrm{~m}$-radius analysers. Currently, $\mathrm{Si}(110)$ and $\mathrm{Si}(111)$ four-crystal $1 \mathrm{~m}$-radius sets are available for users.

\section{Analyser crystals}

We are constantly adding to our portfolio of analyser crystals, which are shown in Table 1 and are updated under the user guide section of the GALAXIES website (https://www.syn chrotron-soleil.fr/fr/lignes-de-lumiere/galaxies). As well as acquiring commercially available analysers, we are able to use anodic bonding for in-house fabrication (Collart et al., 2005). An extensive list of analyser crystals for many absorption and emission energies, together with their energy resolution and reflectivities, has also been published which is useful for planning experiments (Gog et al., 2013). In order to determine the overall energy resolution for an IXS measurement, it is necessary to combine the incident beam resolution [given by the bandwidth of the DCM and/or HRM monochromators (Ablett et al., 2013)], the intrinsic resolution of the analyser crystal as well as other geometrical effects such as the vertical source size contribution and Johann error (Bergmann \&
Table 1

Current X-ray analysers available at GALAXIES and their respective radii of curvature.

The energy ranges correspond to the Bragg angular range of $70^{\circ}$ to $89^{\circ}$. The superscript values in the energy ranges denote the order of reflection. The $\mathrm{Ge}(310), \mathrm{Si}(110)$ and $\mathrm{Si}(111)$ analyser crystals (in bold) are strained-relieved and $*$ indicates a dispersive flat diced analyser.

\begin{tabular}{lll}
\hline Crystal & Radius & Energy range $(\mathrm{eV})$ \\
\hline $\mathrm{Si}(100)$ & $1 \mathrm{~m}$ & $4567 \rightarrow 4859^{4}, 9133 \rightarrow 9718^{8}$ \\
$\mathrm{Si}(\mathbf{1 1 1})$ & $1 \mathrm{~m}$ & $5932 \rightarrow 6312^{3}, 7909 \rightarrow 8416^{4}, 9887 \rightarrow 10520^{5}$ \\
$\mathrm{Si}(\mathbf{1 1 0})$ & $1 \mathrm{~m}$ & $6458 \rightarrow 6871^{4}, 9687 \rightarrow 10307^{6}$ \\
$\mathrm{Si}(311)$ & $1 \mathrm{~m}$ & $3786 \rightarrow 4029^{1}, 11359 \rightarrow 12086^{3}$ \\
$\mathrm{Si}(310)$ & $1 \mathrm{~m}$ & $7220 \rightarrow 7683^{2}$ \\
$\mathrm{Si}(211)^{*}$ & $1 \mathrm{~m}$ & $5593 \rightarrow 5951^{2}, 11186 \rightarrow 11902^{4}$ \\
$\mathrm{Si}(531)$ & $1 \mathrm{~m}$ & $6754 \rightarrow 7186^{1}$ \\
$\mathrm{Si}(533)$ & $2 \mathrm{~m}$ & $7486 \rightarrow 7965^{1}$ \\
$\mathrm{Si}(551)$ & $2 \mathrm{~m}$ & $8153 \rightarrow 8675^{1}$ \\
$\mathrm{Si}(553)$ & $2 \mathrm{~m}$ & $8769 \rightarrow 9330^{1}$ \\
$\mathrm{Ge}(110)$ & $1 \mathrm{~m}$ & $6212 \rightarrow 6610^{4}, 9318 \rightarrow 9914^{6}$ \\
$\mathrm{Ge}(111)$ & $1 \mathrm{~m}$ & $5706 \rightarrow 6071^{3}, 7608 \rightarrow 8095^{4}, 9510 \rightarrow 10119^{5}$ \\
$\mathrm{Ge}(331)$ & $1 \mathrm{~m}$ & $4787 \rightarrow 5093^{1}$ \\
$\mathbf{G e}(\mathbf{3 1 0})$ & $1 \mathrm{~m}$ & $6945 \rightarrow 7390^{2}$ \\
\hline
\end{tabular}

Cramer, 1998). A signifcant improvement in predicting the reflectivity curves for spherical analyser crystals without strain relief, that includes the dominant effect of angular compression due to the analyser bending, has recently been discussed (Honkanen et al., 2014, 2016, 2017).

Fig. 6 shows the estimated total resolution (FWHM) of the GALAXIES spherically bent analyers using their full available area. All geometrical contributions (Bergmann \& Cramer, 1998) and the effect of strain (Honkanen et al., 2014, 2016, 2017) (see Table 1) have been included in the calculation. The highest resolution is obtained by reducing the incident X-ray beam bandwidth [currently using the $\operatorname{Si}(333)$ reflection of the DCM] and using 2 m-radius analyser crystals or analyzer crystals that have been strain relieved (Table 1). The $\mathrm{Si}(110)$ and $\mathrm{Si}(111)$ strain-relieved analyzers are currently used for X-ray Raman spectroscopy at Bragg angles close to 


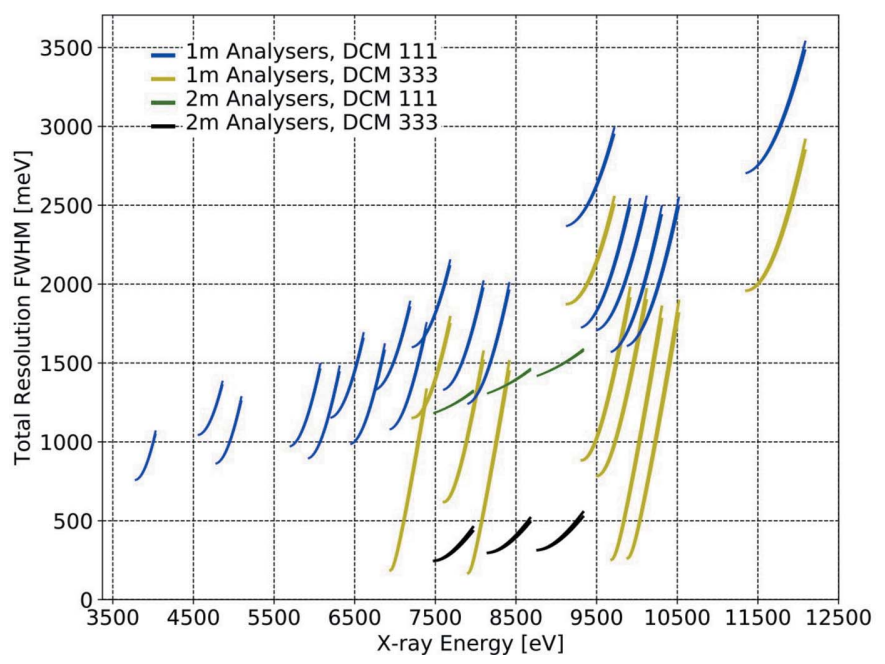

Figure 6

Estimated total resolution of the GALAXIES spherically bent analyzers. The resolution has been estimated by adding in quadrature the incident $\mathrm{X}$-ray beam resolution using either the first- or third-order of the DCM, geometrical effects and analyser stress contributions (where appropiate).

$89^{\circ}$ and achieve $<300 \mathrm{meV}$ FWHM total resolution for these type of measurements.

\section{Sample environments}

We have a wide range of sample environments available for experiments and users can install their own specialized systems. For high-pressure measurements (Ablett et al., 2015; Dorfman et al., 2015, 2016; Baledent et al., 2015; Martin et al., 2016; Baledent \& Rue, 2016), we have several types of gasdriven membrane diamond anvil cells, and a high-pressure laboratory is available for preparing and loading them. A ruby fluorescence laser measuring system is typically mounted within the experimental set-up in order to quickly determine the applied pressure. High-pressure measurements can also be made down to $4 \mathrm{~K}$ with a specially designed helium cryostat (Sundermann et al., 2015; Rueff et al., 2015b) and up to several hundreds of degrees Celsius with a resistive heater. A helium compressor cryostat, for measuring samples down to $15 \mathrm{~K}$, a special oven allowing temperature exceeding $900^{\circ} \mathrm{C}$ and cryostreams are also available. To apply magnetic fields to the sample (Zimmermann et al., 2018), a compact electromagnet with drilled hole pieces that allow the incident X-ray beam to pass can deliver $\pm 0.7 \mathrm{~T}$. A catalytic cell developed in collaboration with the ROCK beamline at SOLEIL and adapted to the RIXS geometry is also available. Finally, an experimental setup for the study of gas phase samples has also been developed (Ismail et al., 2018).

\section{Detectors}

On the X-ray spectrometer detector front, we use either a silicon drift detector (SDD), an avalanche photodiode (APD) or hybrid pixel detectors, depending upon experimental conditions. The fast SDD (SiriusSD, RaySpec, UK) has a $450 \mu \mathrm{m}$ sensor, a collimated area of $80 \mathrm{~mm}^{2}$, a thick $25 \mu \mathrm{m}$ - thick beryllium window and can be used within the entire operating energy range of the end-station. The detector is connected to an XSPRESS mini (Quantum Detectors, UK) fast digital pulse processing unit which can be used up to and above a million counts per second without saturation and incorporates accurate dead-time correction. The high dynamic range APD (FMB Oxford) has a $10 \mathrm{~mm} \times 10 \mathrm{~mm}$ area, a $200 \mu \mathrm{m}$-thick silicon sensor and a $40 \mu \mathrm{m}$ opaque Kapton window. The Merlin pixel (Quantum Detectors, UK) single $(256 \times 256$ pixels $)$ and quad chip $(512 \times 512$ pixels $)$ detectors are also available, for energies above $7 \mathrm{keV}$. These hybrid detectors have $500 \mu \mathrm{m}$-thick sensors with $55 \mu \mathrm{m} \times 55 \mu \mathrm{m}$ size pixels and exhibit a very high dynamic range. One of the important benefits of these detectors is that they can increase the energy resolution when used in conjunction with dispersive analyser systems that are essential when measuring lowenergy excitations (Shvyd'ko, 2004; Huotari et al., 2005, 2006; Ketenoglu et al., 2015). In addition, the ability to define specific 'regions of interest' can help reduce background contributions and can also be used for imaging applications (Huotari et al., 2011).

\section{Continuous on-the-fly scanning}

The incident X-ray energy (combined scanning of the DCM and undulator), the energies of the single- and multi-analyser spectrometers and the sample position stages all use on-the-fly continuous scans. On-the-fly scanning is an essential prerequisite for the operation of the GALAXIES IXS endstation as it enables data collection with no dead-time thus allowing for high data collection throughput. This is particularly advantageous for radiation-sensitive materials. As an example, a HERFD scan around the iron $K$-edge on a $6 \mu \mathrm{m}$ thick Fe foil, using a silicon (531) crystal analyser tuned to the Fe $K \beta$ emission line, with a dwell time of 0.25 s over a $0.3 \mathrm{eV}$ energy interval, can be performed within $90 \mathrm{~s}$ with adequate statistics, which is $\sim 15$ times faster than an equivalent scan performed in step-by-step mode. For these type of scans, the undulator speed is continuously adjusted in order to keep it synchronized with the DCM, and is typically within $1 \mathrm{eV}$ of the undulator maximum intensity throughout the scan. This excellent synchronization is highlighted in Fig. 7 (left) over a $950 \mathrm{eV}$ scan range with a $0.5 \mathrm{~s}$ dwell time over a $0.2 \mathrm{eV}$ energy interval, with a total aquisition time of $\sim 45 \mathrm{~min}$. The diagonal line is the superposition of both the DCM and undulator energies (left axis) and the blue curve is the energy difference between the two (right axis). This energy difference is more than adequate in order to stay on the maximum of the undulator emission as highlighted in Fig. 7 (right).

All on-the-fly scans are defined via commands explicit to the beamline which are based on the Python language. These commands are frequently used in macros in order to automatize the experiment once it has been optimized. A schematic of the control architecture is shown in Fig. 8. The configuration of the hardware is automatically applied once the scan information has been entered by the user. The master clock and controller(s) are simultaneously initiated at the start 

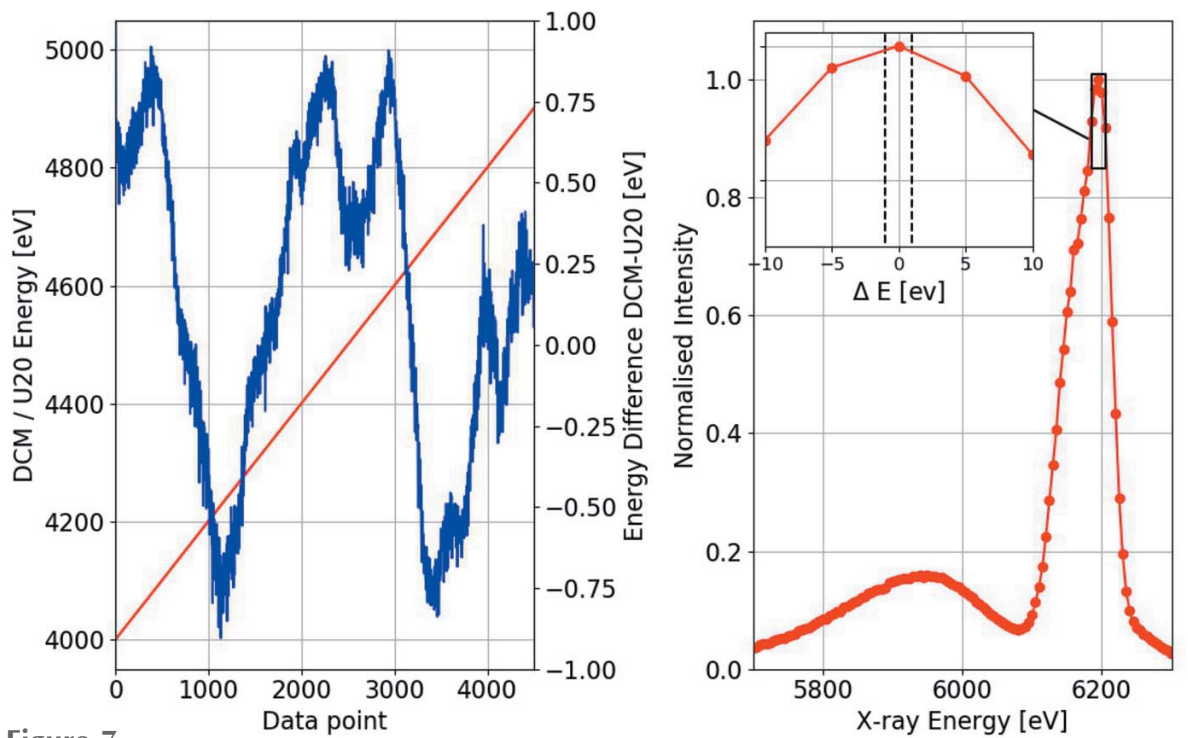

Left: plot showing the excellent synchronization between the DCM and undulator energies during a wide energy scan. The red diagonal line is in fact composed of both the DCM and U20 energies, and the blue curve is the difference between them. Right: profile of the third harmonic (undulator gap = $5.5 \mathrm{~mm}$ ), where the inset shows a zoom-in around the harmonic maximum and the dashed lines around the single data point is the envelope of the difference between the DCM and undulator energies at this position, representing $<0.5 \%$ maximum intensity variation.

of the scan. During a scan, the master clock delivers individual TTL pulses of duration $t$ (the integration time specified by the scan) and separated by a short millisecond delay (which is mostly dictated by the read-out of the detector modules).

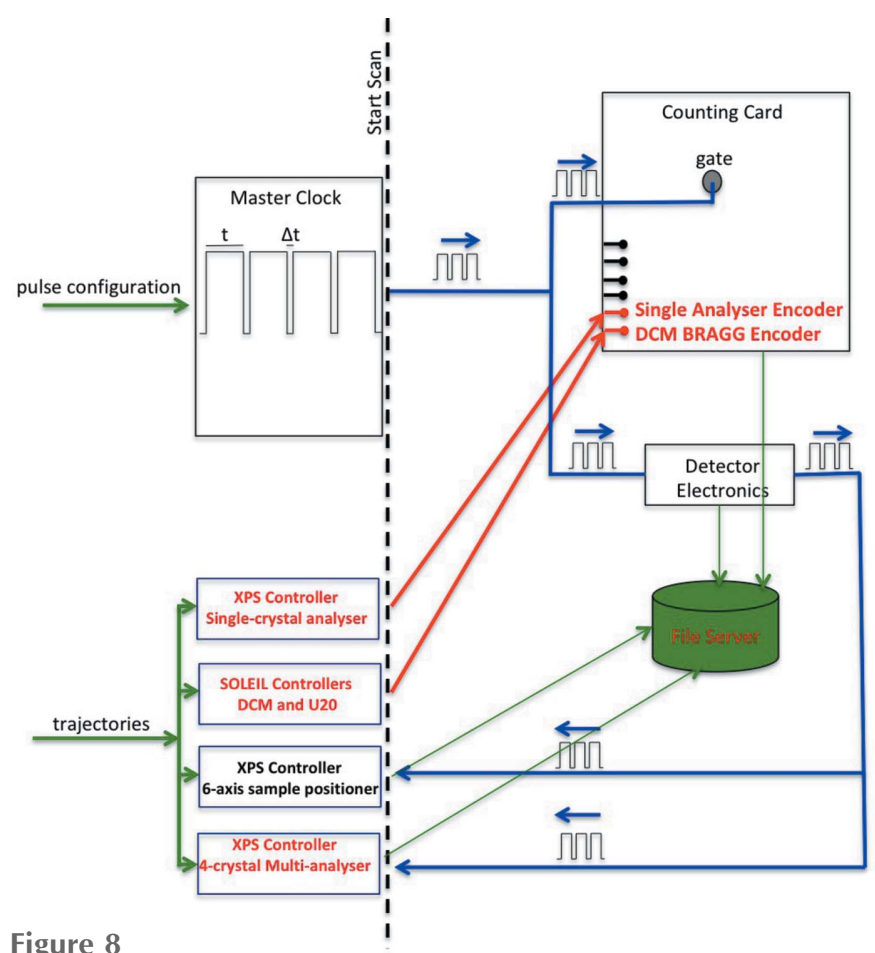

Figure 8

Control system schematic for GALAXIES on-the-fly scanning. These continuous scans are used for scanning both the incident (DCM and undulator) spectrometer energies and also the sample position. The master clock is used to gate the counting card and trigger the detector electronics and Newport XPS controllers. See main text for a more detailed explanation of the system.
These pulses ensure synchronization between the various hardware components during a scan. A counting card, the detector electronics and certain Newport XPS controllers are triggered by these pulses. The counting card, which is gated by the TTL pulses, is used to record the position of the the DCM and single-analyser Bragg angles (and thus their energies). The multi-analyser energy is determined by the position of the height translations of the four analyser motors that are read out synchronously from the XPS controller, in exactly the same manner in which the positions of the six-axis sample stage are determined from a different XPS controller. For the energy scans of the IXS spectrometers and the sample position, the motor trajectories are controlled by the XPS controllers. All data are stored on a server that is labelled 'File Server' in Fig. 8 in both NEXUS and ASCII formats.

An example of on-the-fly scanning is shown in Fig. 9 where X-ray Raman spectroscopy has been used to elucidate the carbon speciation in ancient and historic materials such as fossils and pigments (Gueriau et al., 2017). Fig. 9 shows an XRS spectrum on a few mm-thick graphite sample around the carbon $K$-edge using the third order of four $\mathrm{Si}(111)$ analyser crystals at a Bragg angle of $86^{\circ}$ (incident X-ray energy = $5.9 \mathrm{keV}$ ) in a back-scattering geometry (momentum transfer $\simeq 5.8 \AA^{-1}$ ). The total resolution for this experiment was $0.8 \mathrm{eV}$ FWHM and the plot represents the background

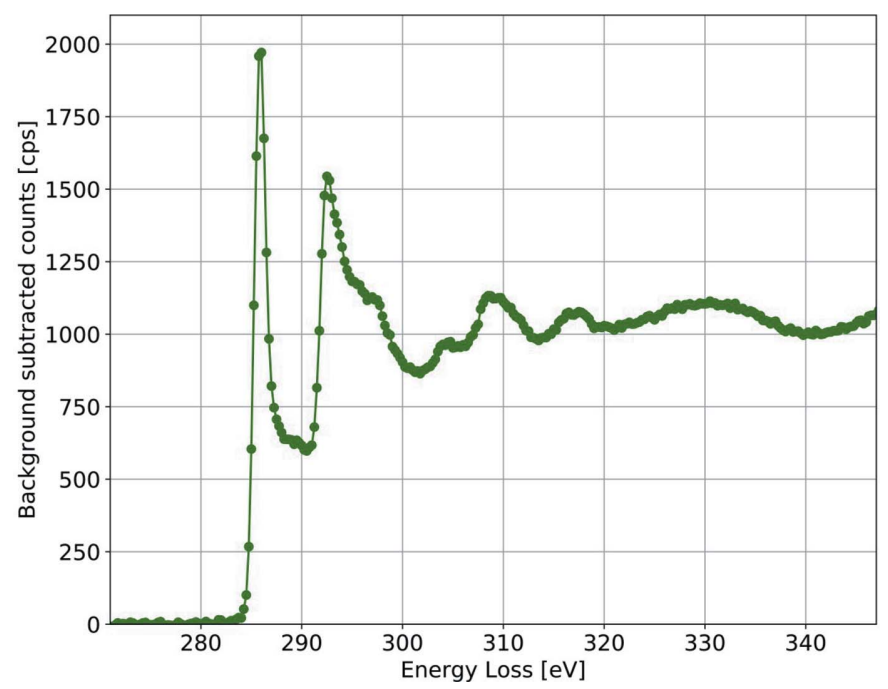

Figure 9

XRS carbon $K$-edge spectra taken on graphite with four $\mathrm{Si}(333)$ analyser crystals set to diffract $5.9 \mathrm{keV}$ X-rays (Bragg angle $86^{\circ}$ ) in a backscattering configuration (momentum transfer of $\sim 5.8 \AA^{-1}$ ). This is an average of 43 XRS scans (background-subtracted) with a total aquisition time of $2.2 \mathrm{~h}$. 
subtracted average of 43 scans with an overall acquisition time of $2.2 \mathrm{~h}$.

\section{Diamond X-ray phase retarder}

Polarization can be controlled in a relatively straightforward manner in the hard X-ray range using XPRs. These devices, which are fabricated from low-absorbing perfect-crystal materials such as diamond or silicon, allow the horizontal polarization of the synchrotron X-ray beam to be transformed into circular, elliptical or vertical polarizations and have long been in operation at numerous hard X-ray synchrotron facilities worldwide (Logan et al., 2016; Hirano \& Maruyama, 1997; Hirano et al., 1993; Giles et al., 1994a,b, 1995; Freeland et al., 2002; Lang \& Srajer, 1995; Strempfer et al., 2013; Paolasini et al., 2007). The GALAXIES XPR mechanical assembly is currently fitted with a $500 \mu \mathrm{m}$-thick single-crystal synthetic diamond (111). The XPR operates in the Laue asymmetric transmission geometry where the [111] reflecting planes subtend an angle of $19.47^{\circ}$ to the XPR [111] surface and is very efficient in terms of transmission and polarization in the energy range from 6 to $9 \mathrm{keV}$. Table 2 shows the important parameters for the XPR.

One area of research where the XPR is currently being exploited is in hard X-ray resonant inelastic X-ray scattering combined with magnetic circular dichroism (RIXS-MCD). This technique allows bulk-sensitive, element- and site-selective magnetic information on complex materials to be obtained. In particular, $1 s 2 p$ RIXS in transition metal compounds, where the $K \alpha(2 p \rightarrow 1 s)$ transition is recorded after resonant excitation of a $1 s$ core electron $(1 s \rightarrow 3 d)$, is of particular interest because the final state in the RIXS process $2 p^{5} 3 d^{n+1}$ is similar to that of the corresponding soft XMCD (Caliebe et al., 1998). The attractiveness of RIXS-MCD is not only in its potential high contrast and high penetrating power of the incident and emitted $\mathrm{X}$-rays but also in the ability to tune the incident X-ray energy to a specific chemical species in mixed valent systems and perform site-selective magnetic hysteresis measurements (Sikora et al., 2012).

The first RIXS-MCD measurements at GALAXIES were performed on $\mathrm{Fe}_{3} \mathrm{O}_{4}$ as a previously measured test example (Sikora et al., 2010). For this experiment, a $1 \mathrm{~m}$-radius $\mathrm{Ge}(440)$ single analyser crystal was used to record the $K \alpha_{1}$ emission line as a function of incident energy, circular polarization (left and right) and magnetic field direction (along the direction of $\mathrm{X}$-ray beam propagation). An electromagnet, with $1.5 \mathrm{~mm}$-diameter holes
Table 2

Parameters for the GALAXIES diamond XPR.

$E$ is the X-ray energy. $\theta_{\mathrm{B}}$ is the Bragg angle. $\omega$ is the angular offset for circular polarization. $t_{\mathrm{eff}}$ and Trans are the effective XPR thickness and corresponding transmission, respectively. $P_{\mathrm{c}}$ is the calculated degree of circular polarization, taking into account the incident X-ray beam divergence.

\begin{tabular}{llllll}
\hline $\begin{array}{l}E \\
(\mathrm{keV})\end{array}$ & $\begin{array}{l}\theta_{\mathrm{B}} \\
\left(^{\circ}\right)\end{array}$ & $\begin{array}{l}\omega \\
(\operatorname{arcsec})\end{array}$ & $\begin{array}{l}t_{\text {eff }} \\
(\mu \mathrm{m})\end{array}$ & $\begin{array}{l}\text { Trans } \\
(\%)\end{array}$ & $\begin{array}{l}P_{\mathrm{c}} \\
(\%)\end{array}$ \\
\hline 6 & 30.08 & 98 & 509 & 14.5 & 99.3 \\
7 & 25.44 & 55 & 502 & 31.5 & 97.9 \\
8 & 22.08 & 33 & 500 & 47 & 94.8 \\
9 & 19.52 & 21 & 500 & 61 & 89.2 \\
\hline
\end{tabular}

drilled through the pole pieces, allowed a magnetic field to be applied to the sample along the direction of the incident $\mathrm{X}$-rays, with a maximum field of $\pm 0.7 \mathrm{~T}$. Fig. 10 shows these results where the upper left-hand plot shows the Fe $K \alpha_{1}$ intensity as a function of incident X-ray energy for both left and right circularly polarized light with an applied field of $+0.7 \mathrm{~T}$ along the incident X-ray beam direction, in the preedge region of the $K$-edge of $\mathrm{Fe}_{3} \mathrm{O}_{4}$. The dramatic strong magnetic contrast of $\sim 16 \%$ in the pre-edge region of the $K$-edge was found, compared with conventional $K$-edge absorption measurements, due to reduced lifetime broadening and increased splitting of the final-state spectral features (Sikora et al., 2010). The difference between these signals for the two magnetic field directions is shown in the bottom left of Fig. 10 and a RIXS-MCD map is displayed on the right-hand side of Fig. 10, showing the dramatic magnetic contrast in the pre-edge region as previously reported (Sikora et al., 2010).
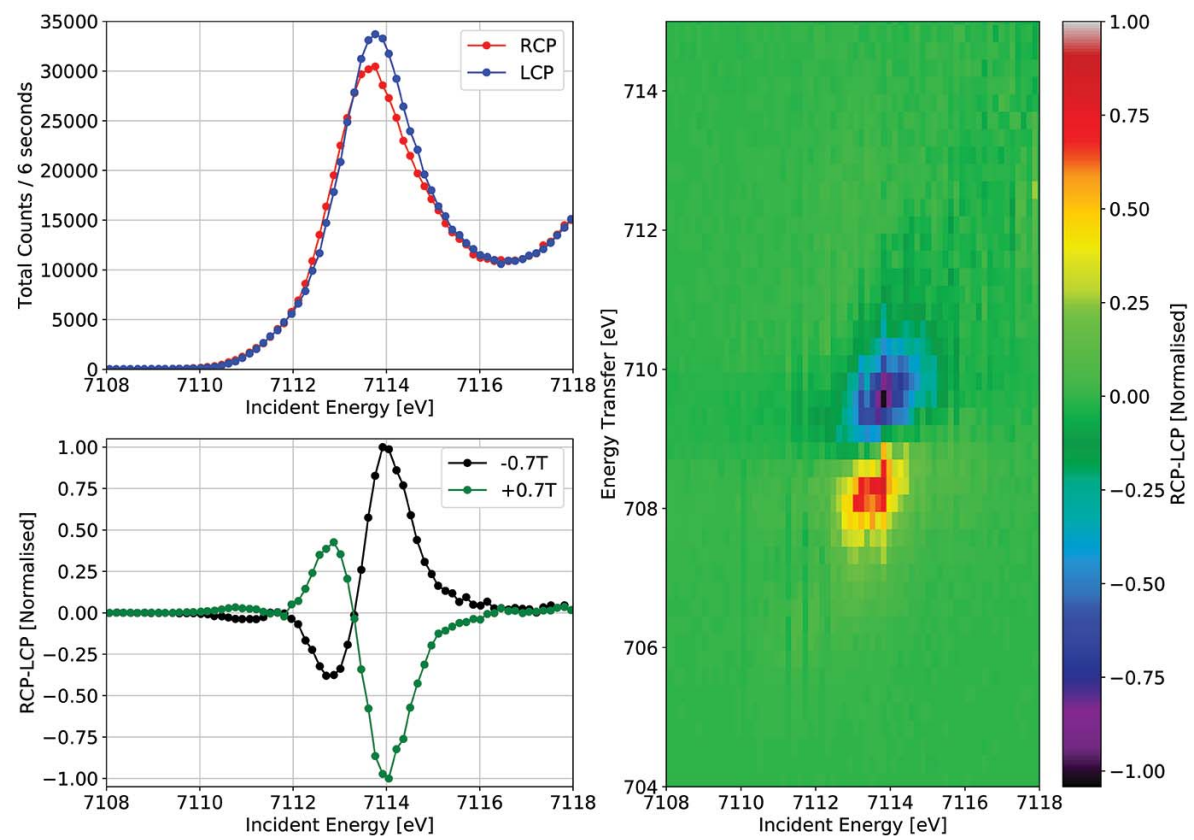

Figure 10

$1 s 2 p$ RIXS-XMCD on $\mathrm{Fe}_{3} \mathrm{O}_{4}$ in the pre-edge region of the $K$-edge. Upper left: $\mathrm{Fe} K \alpha_{1}$ intensity as a function of incident energy for left and right circularly polarized light (LCP and RCP) with the sample magnetized along the incident X-ray direction in a field of $0.7 \mathrm{~T}$. Bottom left: the difference between RCP and LCP Fe $K \alpha_{1}$ intensity as a function of incident X-ray energy for the two magnetic field directions. Right: the RIXS-MCD map showing the dramatic magnetic contrast for RCP-LCP in a magnetic field of $0.7 \mathrm{~T}$. 
We have also recently used $1 s 2 p$ RIXS-MCD in order to determine the magnetic ground state of $\mathrm{CrO}_{2}$ where there is a similar but smaller magnetic contrast compared with magnetite (Zimmermann et al., 2018).

\section{Conclusion}

In this article, we have described and demonstrated the capabilities of the IXS end-station at the GALAXIES beamline. The IXS facility enables a wealth of different experimental techniques to be performed such as X-ray emission, X-ray Raman, resonant- and non-resonant scattering and HERFD spectroscopies. Hard X-ray inelastic scattering is developing into a readily accessible tool for studying the electronic properties of matter at synchrotron radiation facilities. The performance of the facility will be enhanced once the high-resolution monochromator has been commissioned and an upgrade for X-ray Raman spectroscopy comprising a 40-crystal analyser spectrometer is currently underway.

\section{Acknowledgements}

We would like to thank Dr Palyanov and co-workers at the Institute of Geology and Mineralogy of the Russian Academy of Science (Novossibirsk, Russia) for the growth of the synthetic diamond used in the XPR. Much appreciation is given to Professor T. A. Tyson for supplying us with silicon crystals at the start of this project and Dr P. Glatzel from the ESRF for lending us analyser crystals from time to time. We are extremely grateful to Professor A. Juhin and Professor Ph. Sainctavit for help and supplying the sample for the RIXSMCD measurements on magnetite. Finally, we express our gratitude to the various SOLEIL teams including the design, engineering, computing, detector and accelerator groups for their essential support throughout this project.

\section{References}

Ablett, J. M., Dubuisson, J. M., Moreno, T., Céolin, D., Prieur, D., Raimon, E., Corruble, D., Coquet, J., Lestrade, A., Bourgoin, C. \& Rueff, J. P. (2013). J. Phys. Conf. Ser. 425, 052007.

Ablett, J., Rueff, J.-P., Shieh, S., Kao, C. \& Wang, S. (2015). Phys. Rev. $B, 92,014113$.

Alonso-Mori, R., Kern, J., Sokaras, D., Weng, T.-C., Nordlund, D., Tran, R., Montanez, P., Delor, J., Yachandra, V. K., Yano, J. \& Bergmann, U. (2012). Rev. Sci. Instrum. 83, 073114.

Ament, L. J. P., van Veenendaal, M., Devereaux, T. P., Hill, J. P. \& van den Brink, J. (2011). Rev. Mod. Phys. 83, 705-767.

Balédent, V. \& Rueff, J. (2016). High. Press. Res. 36, 371-380.

Balédent, V., Rullier-Albenque, F., Colson, D., Ablett, J. M. \& Rueff, J.-P. (2015). Phys. Rev. Lett. 114, 177001.

Bergmann, U. \& Cramer, S. P. (1998). Proc. SPIE, 3448, 198-209.

Caliebe, W. A., Kao, C. C., Hastings, J. B., Taguchi, M., Kotani, A., Uozumi, T. \& de Groot, F. M. F. (1998). Phys. Rev. B, 58, 1345213458.

Céolin, D., Ablett, J. M., Prieur, D., Moreno, T., Rueff, J. P., Marchenko, T., Journel, L., Guillemin, R., Pilette, B., Marin, T. \& Simon, M. (2013). J. Electron Spectrosc. Relat. Phenom. 190, 188192.
Collart, E., Shukla, A., Gélébart, F., Morand, M., Malgrange, C., Bardou, N., Madouri, A. \& Pelouard, J.-L. (2005). J. Synchrotron Rad. 12, 473-478.

De Groot, F. M. F. \& Kotani, A. (2008). Core Level Spectroscopy of Solids. CRC Press/Taylor \& Francis Group.

Diaz-Moreno, S., Amboage, M., Basham, M., Boada, R., Bricknell, N. E., Cibin, G., Cobb, T. M., Filik, J., Freeman, A., Geraki, K., Gianolio, D., Hayama, S., Ignatyev, K., Keenan, L., Mikulska, I., Mosselmans, J. F. W., Mudd, J. J. \& Parry, S. A. (2018). J. Synchrotron Rad. 25, 998-1009.

Dorfman, S. M., Badro, J., Rueff, J., Chow, P., Xiao, Y. \& Gillet, P. (2015). Am. Mineral. 100, 2246-2253.

Dorfman, S., Dutton, S., Potapkin, V., Chumakov, A., Rueff, J., Chow, P., Xiao, Y., Cava, R., Duffy, T. S., McCammon, C. \& Gillet, P. (2016). Am. Mineral. 101, 1659-1667.

Duan, P., Gu, S., Cao, H., Li, J. \& Huang, Y. (2016). X-ray Spectrom. 46, 12-18.

Fister, T., Seidler, G., Wharton, L., Battle, A., Ellis, T., Cross, J., Macrander, A., Elam, W., Tyson, T. \& Qian, Q. (2006). Rev. Sci. Instrum. 77, 063901.

Freeland, J. W., Lang, J. C., Srajer, G., Winarski, R., Shu, D. \& Mills, D. M. (2002). Rev. Sci. Instrum. 73, 1408-1410.

Gao, X., Gu, S., Gao, Q., Zou, Y., Jiang, Z., Zhang, S., Wei, X., Yu, H., Sheng, G., Duan, P. \& Huang, Y. (2013). X-ray Spectrom. 42, 502507.

Gélebart, F., Morand, M., Dermigny, Q., Giura, P., Rueff, J.-P. \& Shukla, A. (2007). AIP Conf. Proc. 879, 1837-1840.

Giles, C., Malgrange, C., Goulon, J., de Bergevin, F., Vettier, C., Dartyge, E., Fontaine, A., Giorgetti, C. \& Pizzini, S. (1994b). J. Appl. Cryst. 27, 232-240.

Giles, C., Malgrange, C., Goulon, J., de Bergevin, F., Vettier, C., Fontaine, A., Dartyge, E. \& Pizzini, S. (1994a). Nucl. Instrum. Methods Phys. Res. A, 349, 622-625.

Giles, C., Malgrange, C., Goulon, J., de Bergevin, F., Vettier, C., Fontaine, A., Dartyge, E., Pizzini, S., Baudelet, F. \& Freund, A. (1995). Rev. Sci. Instrum. 66, 1549-1553.

Glatzel, P. \& Bergmann, U. (2005). Coord. Chem. Rev. 249, 65-95.

Glatzel, P., de Groot, F. M. F. \& Bergmann, U. (2009b). Synchrotron Radiat. News, 22(2), 12-16.

Glatzel, P., Sikora, M., Smolentsev, G. \& Fernández-García, M. (2009a). Catal. Today, 145, 294-299.

Gog, T., Casa, D. M., Said, A. H., Upton, M. H., Kim, J., Kuzmenko, I., Huang, X. \& Khachatryan, R. (2013). J. Synchrotron Rad. 20, 7479.

Gueriau, P., Rueff, J. P., Bernard, S., Kaddissy, J., Goler, Z., Sahle, C. J., Sokaras, D., Wogelius, R. A., Manning, P. L., Bergmann, U. \& Bertrand, L. (2017). Anal. Chem. 89, 10819-10826.

Hayashi, H. (2013). Encyclopedia of Analytical Chemistry, edited by R. A. Meyers, pp. 1-31. Chichester: John Wiley.

Hazemann, J.-L., Proux, O., Nassif, V., Palancher, H., Lahera, E., Da Silva, C., Braillard, A., Testemale, D., Diot, M.-A., Alliot, I., Del Net, W., Manceau, A., Gélébart, F., Morand, M., Dermigny, Q. \& Shukla, A. (2009). J. Synchrotron Rad. 16, 283-292.

Hirano, K., Ishikawa, T., Nakamura, I., Mizutani, M. \& Kikuta, S. (1993). Jpn. J. Appl. Phys. 33, L689-L692.

Hirano, K. \& Maruyama, H. (1997). Jpn. J. Appl. Phys. 36, L1272L1274.

Honkanen, A.-P., Monaco, G. \& Huotari, S. (2016). J. Appl. Cryst. 49, 1284-1289.

Honkanen, A.-P., Verbeni, R., Simonelli, L., Moretti Sala, M., Monaco, G. \& Huotari, S. (2014). J. Synchrotron Rad. 21, 104-110.

Honkanen, A.-P., Verbeni, R., Simonelli, L., Moretti Sala, M., Monaco, G. \& Huotari, S. (2017). J. Synchrotron Rad. 24, 545546.

Hoszowska, J. \& Dousse, J.-C. (2004). J. Electron Spectrosc. Relat. Phenom. 137-140, 687-690.

Huotari, S., Albergamo, F., Vankó, G., Verbeni, R. \& Monaco, G. (2006). Rev. Sci. Instrum. 77, 053102. 
Huotari, S., Pylkkänen, T., Verbeni, R., Monaco, G. \& Hämäläinen, K. (2011). Nat. Mater. 10, 489-493.

Huotari, S., Sahle, C. J., Henriquet, C., Al-Zein, A., Martel, K., Simonelli, L., Verbeni, R., Gonzalez, H., Lagier, M.-C., Ponchut, C., Moretti Sala, M., Krisch, M. \& Monaco, G. (2017). J. Synchrotron Rad. 24, 521-530.

Huotari, S., Vankó, Gy., Albergamo, F., Ponchut, C., Graafsma, H., Henriquet, C., Verbeni, R. \& Monaco, G. (2005). J. Synchrotron Rad. 12, 467-472.

Ishii, K., Jarrige, J., Yoshida, M., Ikeuchi, K., Inami, T., Murakami, Y. \& Mizuki, J. (2013). J. Electron Spectrosc. Relat. Phenom. 188, 127132.

Ismail, I., Guillemin, R., Marchenko, T., Travnikova, O., Ablett, J., Rueff, J.-P., Piancastelli, M.-N., Simon, M. \& Journel, L. (2018). Rev. Sci. Instrum. 89, 063107.

Ketenoglu, D., Harder, M., Klementiev, K., Upton, M., Taherkhani, M., Spiwek, M., Dill, F.-U., Wille, H.-C. \& Yavaş, H. (2015). J. Synchrotron Rad. 22, 961-967.

Ketenoglu, D., Spiekermann, G., Harder, M., Oz, E., Koz, C., Yagci, M. C., Yilmaz, E., Yin, Z., Sahle, C. J., Detlefs, B. \& Yavaş, H. (2018). J. Synchrotron Rad. 25, 537-542.

Kleymenov, E., van Bokhoven, J., David, C., Glatzel, P., Janousch, M., Alonso-Mori, R., Studer, M., Willimann, M., Bergamaschi, A., Henrich, B. \& Nachtegaal, M. (2011). Rev. Sci. Instrum. 82, 065107. Lang, J. C. \& Srajer, G. (1995). Rev. Sci. Instrum. 66, 1540-1542.

Lelong, G., Cormier, L., Hennet, L., Michel, F., Rueff, J. P., Ablett, J. M. \& Monaco, G. (2017). J. Non-Cryst. Solids, 472, 1-8.

Lelong, G., Radtke, G., Cormier, L., Bricha, H., Rueff, J. P., Ablett, J. M., Cabaret, D., Gélébart, F. \& Shukla, A. (2014). Inorg. Chem. 53, 10903-10908.

Llorens, I., Lahera, E., Delnet, W., Proux, O., Braillard, A., Hazemann, J.-L., Prat, A., Testemale, D., Dermigny, Q., Gelebart, F., Morand, M., Shukla, A., Bardou, N., Ulrich, O., Arnaud, S., Berar, J.-F., Boudet, N., Caillot, B., Chaurand, P., Rose, J., Doelsch, E., Martin, P. \& Solari, P. (2012). Rev. Sci. Instrum. 83, 063104.

Logan, J., Harder, R., Li, L., Haskel, D., Chen, P., Winarski, R., Fuesz, P., Schlagel, D., Vine, D., Benson, C. \& McNulty, I. (2016). J. Synchrotron Rad. 23, 1210-1215.

Martin, N., Deutsch, M., Itié, J., Rueff, J.-P., Rössler, U., Koepernik, K., Fomicheva, L., Tsvyashchenko, A. \& Mirebeau, I. (2016). Phys. Rev. B, 93, 214404.

Moretti Sala, M., Martel, K., Henriquet, C., Al Zein, A., Simonelli, L., Sahle, C., Gonzalez, H., Lagier, M.-C., Ponchut, C., Huotari, S., Verbeni, R., Krisch, M. \& Monaco, G. (2018). J. Synchrotron Rad. 25, 580-591.

Ni, D., Kang, X., Yan, S., Huang, X. C., Xiong, T., Liang, D. X., Yang, K. \& Zhu, L. F. (2018). Rev. Sci. Instrum. 89, 085108.

Paolasini, L., Detlefs, C., Mazzoli, C., Wilkins, S., Deen, P. P., Bombardi, A., Kernavanois, N., de Bergevin, F., Yakhou, F., Valade, J. P., Breslavetz, I., Fondacaro, A., Pepellin, G. \& Bernard, P. (2007). J. Synchrotron Rad. 14, 301-312.
Rovezzi, M. \& Glatzel, P. (2014). Semicond. Sci. Technol. 29, 023002.

Rueff, J.-P. (2009). Magnetism and Synchrotron Radiation, edited by E. Beaurepaire, H. Bulou, F. Scheurer \& J. P. Kappler, Vol. 133 in Springer Proceedings in Physics, pp. 263-277. Springer.

Rueff, J.-P., Ablett, J. M., Céolin, D., Prieur, D., Moreno, T., Balédent, V., Lassalle-Kaiser, B., Rault, J. E., Simon, M. \& Shukla, A. (2015a). J. Synchrotron Rad. 22, 175-179.

Rueff, J.-P., Ablett, J. M., Strigari, F., Deppe, M., Haverkort, M. W., Tjeng, L. H. \& Severing, A. (2015b). Phys. Rev. B, 91, 201108.

Rueff, J.-P. \& Shukla, A. (2010). Rev. Mod. Phys. 82, 847-896.

Rueff, J.-P. \& Shukla, A. (2013). J. Electron Spectrosc. Relat. Phenom. 188, $10-16$.

Schülke, W. (1986). Nucl. Instrum. Methods Phys. Res. A, 246, 491499.

Schülke, W. (2007). Electron Dynamics by Inelastic X-ray Scattering. Oxford University Press.

Shvyd'ko, Y. (2004). X-ray Optics - High-Energy-Resolution Applications. Springer Optical Sciences.

Sikora, M., Juhin, A., Simon, G., Zając, M., Biernacka, K., Kapusta, C., Morellon, L., Ibarra, M. R. \& Glatzel, P. (2012). J. Appl. Phys. 111, 07E301.

Sikora, M., Juhin, A., Weng, T. C., Sainctavit, P., Detlefs, C., de Groot, F. M. F. \& Glatzel, P. (2010). Phys. Rev. Lett. 105, 037202.

Sokaras, D., Nordlund, D., Weng, T.-C., Mori, R. A., Velikov, P., Wenger, D., Garachtchenko, A., George, M., Borzenets, V., Johnson, B., Qian, Q., Rabedeau, T. \& Bergmann, U. (2012). Rev. Sci. Instrum. 83, 043112.

Sokaras, D., Weng, T.-C., Nordlund, D., Alonso-Mori, R., Velikov, P., Wenger, D., Garachtchenko, A., George, M., Borzenets, V., Johnson, B., Rabedeau, T. \& Bergmann, U. (2013). Rev. Sci. Instrum. 84, 053102.

Strempfer, J., Francoual, S., Reuther, D., Shukla, D. K., Skaugen, A., Schulte-Schrepping, H., Kracht, T. \& Franz, H. (2013). J. Synchrotron Rad. 20, 541-549.

Sundermann, M., Chen, K., Yavaş, H., Lee, H., Fisk, Z., Haverkort, M., Tjeng, L. \& Severing, A. (2017). Europhys. Lett. 117, 17003.

Sundermann, M., Strigari, F., Willers, T., Winkler, H., Prokofiev, A., Ablett, J. M., Rueff, J.-P., Schmitz, D., Weschke, E., Sala, M. M., Al-Zein, A., Tanaka, A., Haverkort, M. W., Kasinathan, D., Tjeng, L. H., Paschen, S. \& Severing, A. (2015). Sci. Rep. 5, 17937.

Szlachetko, J., Nachtegaal, M., Grolimund, D., Knopp, G., Peredkov, P., Czapla-Masztafiak, J. \& Milne, C. (2017). Appl. Sci. 7, 899.

Veenendaal, M. van (2014). Theory of Inelastic Scattering of X-rays. Cambridge University Press.

Verbeni, R., Pylkkänen, T., Huotari, S., Simonelli, L., Vankó, G., Martel, K., Henriquet, C. \& Monaco, G. (2009). J. Synchrotron Rad. 16, 469-476.

Xiao, Y., Chow, P. \& Shen, G. (2016). High. Press. Res. 36, 315-331.

Zimmermann, P., Bouldi, N., Hunault, M. O. J. Y., Sikora, M., Ablett, J. M., Rueff, J.-P., Lebert, B., Sainctavit, P., de Groot, F. M. F. \& Juhin, A. (2018). J. Electron Spectrosc. Relat. Phenom. 222, 74-87. 